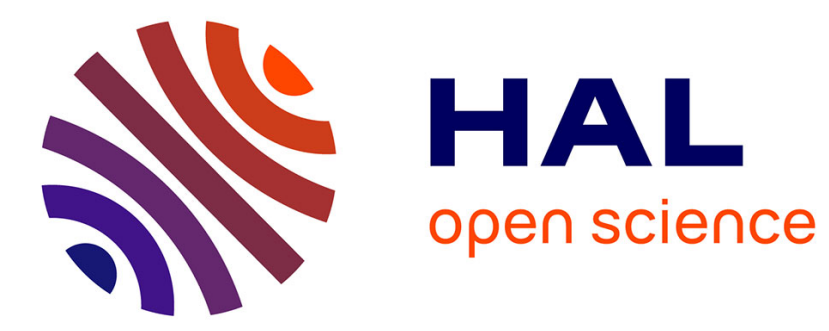

\title{
High negative charge of a dust particle in a hot cathode discharge
}

Cécile Arnas, Maxime Mikikian, Fabrice Doveil

\section{To cite this version:}

Cécile Arnas, Maxime Mikikian, Fabrice Doveil. High negative charge of a dust particle in a hot cathode discharge. Physical Review E : Statistical, Nonlinear, and Soft Matter Physics, 1999, 60 (6), pp.7420. 10.1103/PhysRevE.60.7420 . hal-00432074

\section{HAL Id: hal-00432074 https://hal.science/hal-00432074}

Submitted on 18 Nov 2009

HAL is a multi-disciplinary open access archive for the deposit and dissemination of scientific research documents, whether they are published or not. The documents may come from teaching and research institutions in France or abroad, or from public or private research centers.
L'archive ouverte pluridisciplinaire HAL, est destinée au dépôt et à la diffusion de documents scientifiques de niveau recherche, publiés ou non, émanant des établissements d'enseignement et de recherche français ou étrangers, des laboratoires publics ou privés. 


\title{
High negative charge of a dust particle in a hot cathode discharge
}

\author{
C. Arnas, M. Mikikian, F. Doveil \\ Equipe Turbulence Plasma - Laboratoire de Physique des Interactions Ioniques et Moléculaires, \\ UMR 6633 - CNRS/Univ. de Provence, Centre de Saint Jérôme, case 321, \\ Avenue Escadrille Normandie Niemen, 13397 Marseille Cedex 20, France
}

\begin{abstract}
Dust particle levitation experiments in a plasma produced by a hot filament discharge, operating at low argon pressure, are presented. The basic characteristics of a dust grain trapped in a plate sheath edge in these experimental conditions are reported. Taking into account the sheath potential profiles measured with a differential emissive probe diagnostic, the forces applied to an isolated dust grain can be determined. Two different experimental methods yield approximately the same value for the dust charge. The observed high negative charge is mainly due to the contribution of the primary electrons emitted by the filaments as predicted by a simple model.
\end{abstract}

\section{INTRODUCTION}

Dust particles trapped in a laboratory plasma can acquire from a few to several thousand electron charges depending on their size and on plasma conditions. In the case of high dust concentration (rf discharge for plasma processing or in dc glow discharge) several plasma properties can be drastically modified: the charge equilibrium [1-2], the plasma particles collective behavior [3-8] and the plasma particles transport [9-10]. Moreover, crystallization [11-17] of dust grains can be observed [18-25]. In symmetrical parallel-plate rf discharges, dust particles of small size $(<0.1 \mu \mathrm{m})$ can accumulate in the center of the electropositive plasma [26-27], in local maxima of the plasma potential [28], in the plasma-sheath transition of the upper electrode [29] or of the lower electrode [30]. Large dust particles are usually trapped at the sheath edge of the lower electrode [18-24]. In general, their location is determined by the relative strength of the forces applied to them due to gravity, electric fields, ion and gas drag and in certain conditions, thermophoresis effects [31-32].

We report experiments on dust particle levitation in the sheath edge of a conducting horizontal disc plate embedded in a continuous argon discharge plasma. Permanent magnets fixed on the outside device wall (magnetic box) enable us to operate at low argon pressure: $\mathrm{P}_{\mathrm{ar}}=10^{-3} \mathrm{mbar}$ (colisionless plasma). The ionization sources are two heated tungsten filaments emitting energetic primary electrons. This population is correctly represented by an isotropic drifting Maxwellian distribution. In these conditions, using the Orbital Motion Limited (OML) model [33], we show that the main contribution to the high negative charge of an isolated dust particle is due to the primary electrons.

Using a differential emissive probe diagnostic, we measure the sheath-presheath profile of the disc plate, for different negative plate biases. Taking into account the shape of the obtained potential distributions and the dust charge given by the OML model, we can evaluate the strength of the forces applied to a dust grain. Because we use dust particles with relatively large radius, only the gravitation and the electric forces play a significant role.

The dust charge is evaluated experimentally by two methods. The first one consists in measuring the levitation height $h$. When the negative plate voltage is increased, $h$ increases too. For any plate bias, the equilibrium height corresponds to the same sheath potential (and the same electric field) where the balance between the electric and the gravitation forces is achieved. The second one is provided by natural vertical oscillations exhibited by a dust particle around its equilibrium position, in the measured potential profile. The charges found in both ways are compared to the value predicted by the OML model.

\section{EXPERIMENTAL SET UP}

\section{A. Apparatus}

The experiments are performed in a multipolar stainless-steel cylinder, $40 \mathrm{~cm}$ long and $30 \mathrm{~cm}$ in diameter very similar to the one presented in Ref. [34]. The ionization sources are two heated tungsten filaments (shaped like springs with $20 \mathrm{~cm}$ length) situated at the bottom of the device as sketched on Fig. 1. Each filament is heated by a current delivered by a current regulated power supply. The primary electrons emitted by the hot filaments are accelerated towards the grounded wall by the negative discharge voltage $V_{D}=-40 \mathrm{~V}$, provided by a voltage regulated power supply, the plasma discharge appearing at $\mathrm{V}_{\mathrm{D} 0}=-35 \mathrm{~V}$. The discharge current $\mathrm{I}_{\mathrm{d}} \sim 0.16 \mathrm{~A}$ is detected as a voltage drop across a resistor of $1 \Omega$ in series with the discharge circuit. Permanent magnets with alternate polarity are fixed outside the device to provide a magnetic confinement of the primary electrons. This technique allows us to work at low argon pressure: $\mathrm{P}_{\mathrm{ar}}=10^{-3}$ mbar where the ionization mean free path is of the order of $200 \mathrm{~cm}$. So, the plasma produced in this way is collisionless and Langmuir probe measurements give an ion (electron) density of the order of $10^{9} \mathrm{~cm}^{-3}$. In the center of the 


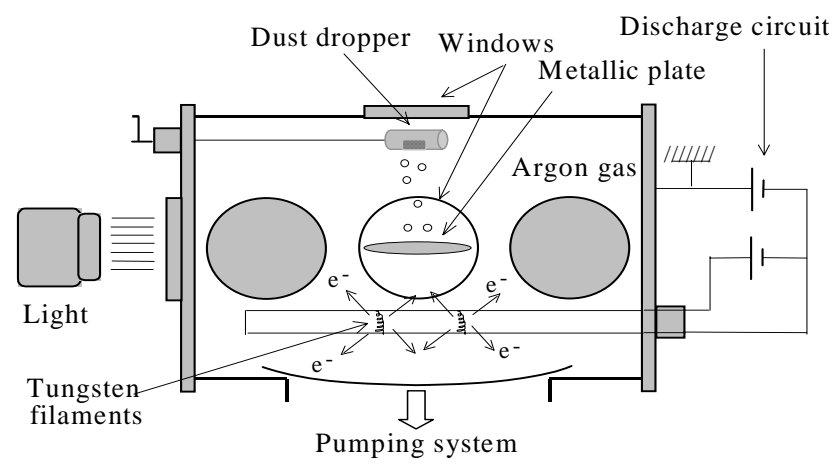

Fig. 1: Multipolar device in series with the continuous discharge circuit. A conducting disc plate over which dust particles levitate is set in the center of the chamber.

device, we have set a stainless steel horizontal disc plate, $12 \mathrm{~cm}$ in diameter. This plate can be left to the negative floating potential or can be more negatively biased using an external power supply. A dust dropper can be driven above the plate and removed when dust grains are trapped in the plate sheath edge. The dust particles are made of hollow glass microspheres of radius $r_{d}=(32 \pm 2) \mu m$ with a mass density $\rho_{d} \sim 110$ $\mathrm{kg} / \mathrm{m}^{3}$. To observe them, they are illuminated with white light and a CCD camera records their horizontal and vertical trajectories. In these conditions, the dust particles are trapped at the plate sheath edge in a horizontal plane, parallel to the plate.

\section{B. Differential emissive probe}

The differential emissive probe diagnostic proposed by En Yao et al. [35] to measure the plasma potential as well as the small potential variations in what is usually called the presheath, consists of two heated identical Langmuir probes. The first one operates without emission and the second one with electron emission. A feedback control circuit applied to this system gives the local potential corresponding to the first common point when both probe characteristics are superposed. Indeed, this point corresponds to the beginning of the electron saturated current where the difference between the two probes currents is zero. With this diagnostic, the spatial resolution is limited by the size of the wires and their separation. We have used two identical tungsten filaments obtained from commercial light bulbs. The spatial resolution cannot be lower than $1 \mathrm{~mm}$. Each end of both filaments is connected to 4 copper wires inserted into four small ducts drilled into an insulator ceramic tube, $3 \mathrm{~mm}$ in diameter, and connected to the external control circuit through a flange. This probe diagnostic can be moved perpendicularly to the plate.

\section{DUST PARTICLE CHARGE GIVEN BY THE ORBITAL MOTION LIMITED MODEL}

In low temperature laboratory plasmas, an isolated dust grain acquires a negative charge when the balance of the ion and electron currents on its surface is achieved. The charge can be predicted in a reasonable approximation by the so-called 'Orbital Motion Limited" model [33] where the dust grain is considered as a spherical probe and must fulfill the condition: $r_{d}<<\lambda_{D}, \lambda_{D}$ being the effective Debye length. The currents are calculated by assuming that the electrons and ions are collected if their collisionless orbits (collisionless plasma) intersect the probe surface. In a multipolar continuous discharge in which hot filaments are used as cathodes, the currents that contribute to the charging process must include the primary electron contribution [36]. Depending on their energy, they can induce secondary electron emission when they impact the dust surface. So, the net charge of an isolated dust in the considered experimental conditions must fulfill the following equation:

$$
I_{e}(\phi)+I_{p e}(\phi)+I_{i}(\phi)=0
$$

where $I_{e}, I_{p e}$ and $I_{i}$ are respectively the electron, the primary electron and the ion currents on the dust grain surface. $\phi=V_{d}-V$ is the difference between the floating potential of the dust grain $V_{d}$ and the local potential. The velocity distribution of the background plasma electrons is Maxwellian with a temperature $T_{e}$ (checked experimentally with Langmuir probe characteristics). The corresponding current onto the dust particle is given by:

$$
I_{e}(\phi)=\operatorname{Sen}_{e} \sqrt{\frac{k T_{e}}{2 \pi m_{e}}} \exp \left(\frac{e \phi}{k T_{e}}\right)
$$

where $\mathrm{S}$ is the dust grain surface, e is the electron charge and $\mathrm{n}_{\mathrm{e}}$ is the electron density.

In our experimental conditions, the primary electrons population is correctly modeled by an isotropic drifting Maxwellian function with a drift velocity $\mathrm{v}_{\mathrm{b}}$ corresponding to the drift energy: $\mathrm{E}_{\mathrm{b}}=\mathrm{m}_{\mathrm{e}}$ $\mathrm{v}_{\mathrm{b}}{ }^{2} / 2$. Indeed, the Langmuir probe characteristics are well "fitted" by the sum of two currents resulting from two distribution functions, the first one centered at the energy corresponding to the plasma potential (background plasma electrons) and the second one whose maximum is shifted to the drift energy $E_{b}$. This result differs from what Walch et al. [36] have observed in a multipolar device operating at an argon pressure $\mathrm{P}_{\mathrm{ar}}=2.610^{-6} \mathrm{mbar}$ and a discharge voltage $\mathrm{V}_{\mathrm{D}}=-60 \mathrm{~V}$. In their case, because of a large ionization collision mean free path (about $510^{5}$ $\mathrm{cm}$ ), the primary electron beam emitted from the filaments remains quasi-monoenergetic. At the pressure $\mathrm{P}_{\mathrm{ar}}=10^{-3}$ mbar used in our experiment, the inelastic collisions with neutrals are greatly increased (ionization mean free path of the order of $200 \mathrm{~cm}$ ). Moreover, the voltage drop across the filaments length introduces an initial spread of the primary electrons energy. These facts could explain the observed spread of the distribution function of this population. With the 
use of an isotropic drifting Maxwellian distribution function, we have calculated the corresponding primary electron current given by:

$$
\begin{aligned}
I_{p e}= & \frac{\operatorname{Sen}_{p e}}{2 \sqrt{\pi}}\left\{v_{p e}\left(x_{b} x_{p}+1\right) e^{-x_{m}^{2}}\right. \\
& \left.+\sqrt{\pi} v_{b}\left(x_{m} x_{p}+\frac{3}{2}\right)\left[1+\operatorname{erf}\left(x_{m}\right)\right]\right\}(1-\delta)
\end{aligned}
$$

where $n_{p e}, T_{p e}$ and $v_{p e}$ are respectively, the density, temperature and thermal velocity of the primary electrons, $x_{b}=v_{b} / v_{p e}, \quad x_{p}=\left(v_{b}+v_{m}\right) / v_{p e}$ where $\mathrm{v}_{\mathrm{m}}=\left(2 e \phi / m_{e}\right)^{1 / 2}$ and $\mathrm{x}_{\mathrm{m}}=\left(\mathrm{v}_{\mathrm{b}}-\mathrm{v}_{\mathrm{m}}\right) / v_{p e} . \delta$ is the coefficient of secondary electron emission. It depends on the dust material and on the primary electron energy. Its variation in the case of a Maxwellian distribution function is given in Ref. [37].

For the ion current, we consider that a dust particle at the sheath edge of a negative plate is charged by monoenergetic ions flowing from the background plasma to the plate. Assuming that the ions enter the sheath at the sound velocity $\mathrm{c}_{\mathrm{s}}=\left(\mathrm{kT}_{\mathrm{e}} / \mathrm{m}_{\mathrm{i}}\right)^{1 / 2}$ (Bohm's criterion), when they hit the dust particle their velocity $\mathrm{v}_{\mathrm{i}}$ is given by energy conservation: $\left(m_{i} v_{i}^{2}\right) / 2=\left(m_{i} c_{s}^{2}\right) / 2-e \phi$, where $\mathrm{m}_{\mathrm{i}}$ is the ion mass. The conservation of angular momentum gives: $v_{i} r_{d}=c_{s} b_{c}$ where $b_{c}$ is the collection impact parameter for an ion grazing the dust particle. Combining both relations, we deduce:

$$
b_{c}=r_{d}\left[1-2 e \phi / m_{i} v_{i}^{2}\right]^{1 / 2}
$$

Then, the ion charging current is:

$$
\mathrm{I}_{\mathrm{i}}(\phi)=-\pi b_{c}^{2} e n_{i} c_{s}=-\frac{S}{4} e n_{i} c_{s}\left(1-\frac{2 \mathrm{e} \phi}{\mathrm{m}_{\mathrm{i}} c_{s}^{2}}\right)
$$

where $n_{i}$ is the ion density.

The charge $\mathrm{Q}_{\mathrm{d}}$ of a dust particle is determined by: $\mathrm{Q}_{\mathrm{d}}=\mathrm{CV}_{\mathrm{d}}$ where $\mathrm{C}=4 \pi \varepsilon_{0} \mathrm{r}_{\mathrm{d}}$ is the capacitance of a sphere with radius $r_{d}$ [38]. In a typical situation where $\mathrm{n}_{\mathrm{e}}=710^{8} \mathrm{~cm}^{-3}, \mathrm{~T}_{\mathrm{e}}=2.5 \mathrm{eV}, \mathrm{n}_{\mathrm{pe}}=310^{7} \mathrm{~cm}^{-3}$, $\mathrm{T}_{\mathrm{pe}}=0.8 \mathrm{eV}$ and $\mathrm{E}_{\mathrm{b}}=19 \mathrm{eV}$, the measured plasma potential is $\mathrm{V}_{\mathrm{p}}=-1.7 \mathrm{~V}$. If we take for the local potential $V_{p}$, the potential for which Eq. (1) is fulfilled is then: $V_{d}=-17 \mathrm{~V}$. This quite high negative value is mainly due to the contribution of the primary electrons and provides, for $r_{d}=(32 \pm 2)$ $\mu \mathrm{m}$, the high negative dust grain charge: $\left(\mathrm{Q}_{\mathrm{d}}\right)_{\mathrm{OML}}=$ $(3.7 \pm 0.3) 10^{5} \mathrm{e}^{-}$.

\section{FORCES ACTING ON A DUST PARTICLE}

\section{A. Gravitation force}

The first force, which affects the dust particle motion, is due to gravity. The magnitude of this force is given by:

$$
\mathrm{F}_{\mathrm{g}}=\mathrm{m}_{\mathrm{d}} \mathrm{g}=\frac{4}{3} \pi r_{d}^{3} \rho_{d} \mathrm{~g}
$$

where $m_{d}$ is the mass of the dust particle, $g$ is the gravity acceleration. For the kind of particles we consider with $r_{d}=(32 \pm 2) \mu \mathrm{m}, \mathrm{Fg}=(1.5 \pm 0.3) 10^{-10} \mathrm{~N}$.

\section{B. Electric force}

This force is exerted by the electric field E perpendicular to the plate. It is given by:

$$
F_{e}=Q_{d} E=-4 \pi \varepsilon_{0} r_{d} V_{d} \frac{\partial V}{\partial z}
$$

where $\mathrm{V}$ is the local sheath potential.

\section{Ion drag force}

The ion flow, which is constant perpendicularly to the plate sheath, exerts a force having two components: the collection and the orbit drag forces [9]. In the case of the collection force, it is considered that all the ions impinging a dust grain transfer their momentum to it. The collection force is represented by:

$$
F_{c o l}=n_{i} \mu v_{i}^{2}\left(\pi b_{c}^{2}\right)
$$

where $\mu$, the reduced mass of the system dust-ion in the laboratory frame, is equal to the ion mass and $b_{c}$ is the collection impact parameter defined in Eq. (4).

Using again the conservation laws of energy and angular momentum, the Coulomb drag force is:

$$
\mathrm{F}_{\text {coul }}=n_{i} \mu v_{i}^{2}\left(4 \pi b_{\pi / 2}^{2}\right) \operatorname{Ln} \Lambda
$$

where the impact parameter at right angle is: $b_{\pi / 2}=e Q_{d} /\left(4 \pi \varepsilon_{o} m_{i} v_{i}^{2}\right)$ and $\operatorname{Ln} \Lambda$ is the Coulomb logarithm integrated over the interval from $b_{c}$ to $\lambda_{\mathrm{D}}: \operatorname{Ln} \Lambda=\operatorname{Ln}\left(\left(\lambda_{D}^{2}+b_{\pi / 2}^{2}\right) /\left(b_{c}^{2}+b_{\pi / 2}^{2}\right)\right)$.

Because of the low working pressure (molecular regime) and because there is no gas flux, the gas drag force is negligible.

For a given dust charge, the electric force depends on the local potential $\mathrm{V}$. The ion drag forces also depend on $\mathrm{V}$ through the impact parameter $b_{c}$, the ion density $n_{i}$ and velocity $v_{i}$ (their variations are given by the energy and momentum conservation laws). Therefore, to find the potential $\mathrm{V}$ for which the forces are balanced, the sheath potential profile must be known. 


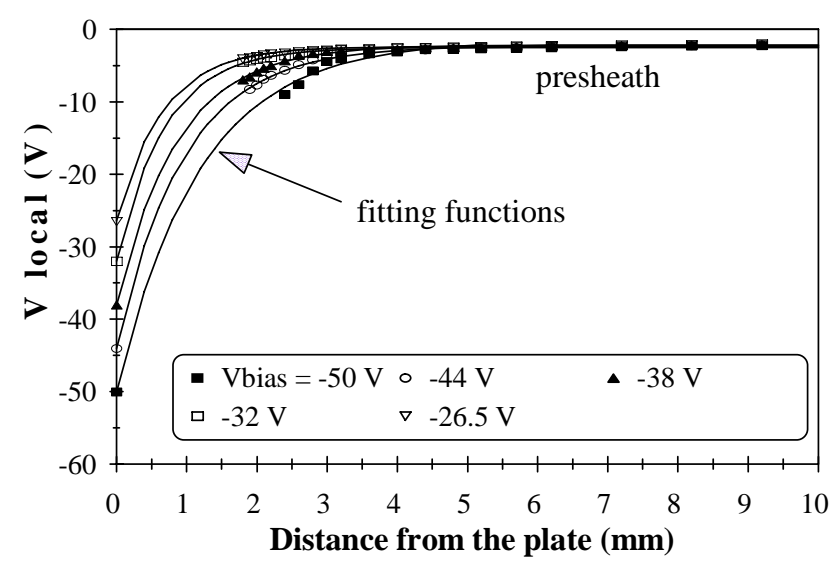

Fig. 2: Plate sheath-presheath vertical potential profiles, for five different plate biases. The symbols represent the measurements made with a differential emissive probe diagnostic and the curves are the best fits given by Eq. (10). The plate biases are the following: floating potential $-26.5 \mathrm{~V}$ and applied voltages: $-32,-38,-44$ and $-50 \mathrm{~V}$.

\section{DETERMINATION OF THE FORCES ACTING ON AN ISOLATED DUST AND THE DUST CHARGE FROM THE PLATE SHEATH AND PRESHEATH POTENTIAL MEASUREMENTS}

The differential emissive probe system can provide the sheath-presheath profile, in a direction perpendicular to the plate. Figure 2 gives different profiles as a function of the distance to the plate in $\mathrm{mm}$, obtained for five plate biases: the floating potential $-26.5 \mathrm{~V}$ and for $-32,-38,-44$ and $-50 \mathrm{~V}$, driven by an external power supply. The plate is at position 0 and the reported data stop at $10 \mathrm{~mm}$. As we see, the effect of increasing the plate voltage is to shift the potential distribution toward the plasma. The plasma potential is reached at about $20 \mathrm{~mm}$. Each set of measurement can be well fitted by a function:

$$
\mathrm{V}(\mathrm{z})=-\mathrm{a} \cdot \exp (-\mathrm{b} . \mathrm{z})+\mathrm{c}
$$

represented by the curves in the diagram of Fig. 2, where $\mathrm{z}$ is the vertical direction. The parameters $\mathrm{a}$ and $\mathrm{b}$ depend on the plate voltage and c has a common value equal to the plasma potential $(-1.7 \mathrm{~V})$.

Using the empirical function (10) and assuming that the presence of a dust particle does not significantly alter the local potential profile, we can now calculate the forces applied on an isolated dust particle. In the case where the plate is left, for example, at the floating potential, Fig. 3 gives the intensity of the forces as a function of the distance to the plate, in $\mathrm{mm}$. The electric and ion drag forces are calculated with the charge $\left(\mathrm{Q}_{\mathrm{d}}\right)_{\mathrm{OML}}$ $=(3.7 \pm 0.3) 10^{5} \mathrm{e}^{-}$. The ion collection drag force is totally negligible and the ratio between the gravitation force and the ion coulomb drag force is of the order of 15 , these results being the consequence of the large

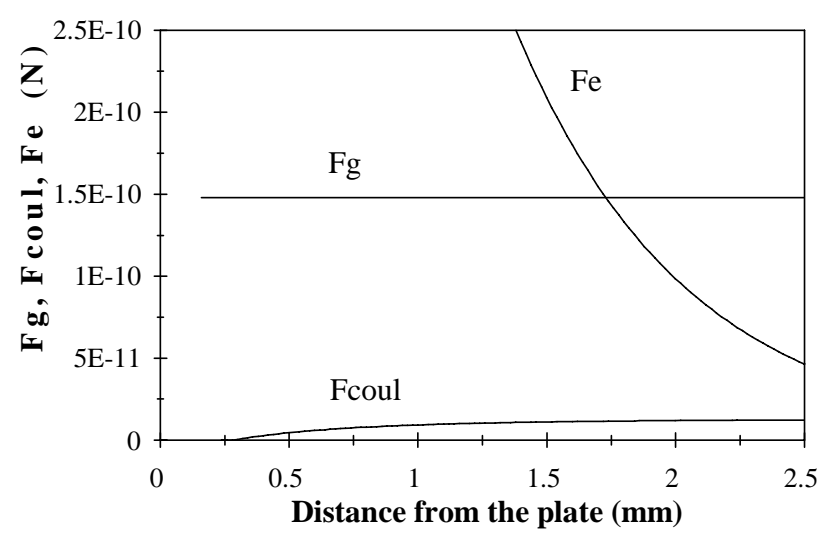

Fig. 3: Forces applied to a dust particle $\left(r_{d}=32 \mu \mathrm{m}\right)$ versus the distance to the plate in $\mathrm{mm}$, in the case of a plate at floating potential. The collection ion force is negligible and the Coulomb drag force is small enough so that the electric force $\mathrm{F}_{\mathrm{e}}$ only balances the gravitation force $\mathrm{F}_{\mathrm{g}}$.

dust radius. So, we can reasonably conclude that the equilibrium position of the dust is simply given by the balance of the electric force $F_{e}$ and the gravitation force $\mathrm{F}_{\mathrm{g}}$.

The charge can thus be estimated by measuring the levitation height. Using the monotonous function (10), the distance from the plate gives directly the sheath potential where the forces are balanced. Because we can inject a unique dust particle in the plasma, we can study its position change over the plate. In Fig. 4, five CCD camera frames are superimposed, each one showing the position of the same levitating isolated dust grain for a given plate voltage. We observe that when the plate bias is increased, the levitation height rises. For each $\mathrm{V}_{\text {bias, }}$, we have reported in Table 1 the measured levitation height $h_{\text {exp. }}$. Each one corresponds to almost the same equilibrium sheath potential $\mathrm{V}_{\text {eq }}$ and local electric field $\mathrm{E}_{\mathrm{eq}}$. For each case, the dust charge found by writing $F_{e}=F_{g}$ is calculated and provides approximately the same value. So, we

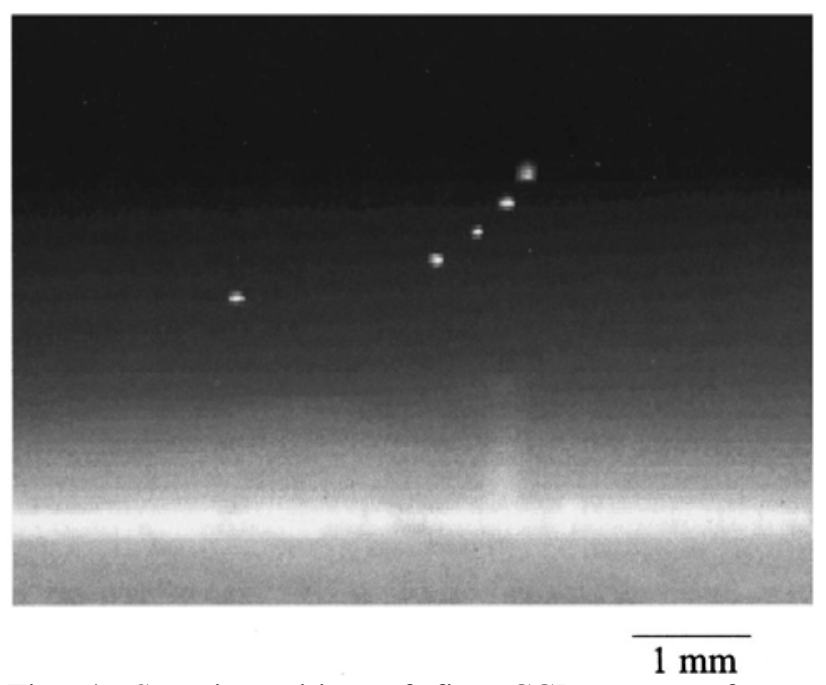

Fig. 4: Superimposition of five CCD camera frames showing the levitation height increase of the same dust particle above a conducting plate (at the bottom of the figure) when the plate negative voltage is increased from $-26.5 \mathrm{~V}$ to $-32,-38,-44$ and $-50 \mathrm{~V}$. 
Table 1: For each plate bias: $-26.5,-32,-38,-44$ and $-50 \mathrm{~V}$, the measurement of a dust levitation height $h_{\exp }$ is given. The corresponding sheath potential $\mathrm{V}_{\text {eq }}$ (and electric field $\left.E_{e q}\right)$ for which the electric force $F_{e}$ balances the gravitation force $F_{g}$ is nearly constant. The corresponding dust particle charge $\mathrm{Q}_{\mathrm{d}}$ is also nearly constant. The mean value $\mathrm{V}_{\text {eq }}=($ $-4.01 \pm 0.12) \mathrm{V}$ provides the mean dust particle charge: $\mathrm{Q}_{\mathrm{d}}=(4.4 \pm 1.2) 10^{5} \mathrm{e}^{-}$.

\begin{tabular}{|c|c|c|c|c|c|}
\hline $\mathbf{V}_{\text {bias }}(\mathbf{V})$ & $\mathbf{- 2 6 . 5}$ & $-\mathbf{3 2}$ & $\mathbf{- 3 8}$ & $\mathbf{- 4 4}$ & $\mathbf{- 5 0}$ \\
\hline $\mathbf{h}_{\mathbf{e x p}}(\mathbf{m m})$ & 1.90 & 2.33 & 2.62 & 2.90 & 3.34 \\
\hline $\mathbf{V}_{\text {eq }}(\mathbf{V})$ & -3.88 & -3.60 & -3.89 & -4.25 & -4.40 \\
\hline $\mathbf{E}_{\text {eq }}(\mathbf{k V} / \mathbf{m})$ & 2.11 & 1.62 & 2.11 & 2.17 & 2.40 \\
\hline $\mathbf{Q}_{\mathbf{d}}\left(\mathbf{1 0}^{\mathbf{5}} \mathbf{e}^{-}\right)$ & 4.3 & 5.6 & 4.3 & 4.2 & 3.8 \\
\hline
\end{tabular}

conclude that an isolated dust particle of radius 32 $\mu \mathrm{m}$ is in equilibrium in the plate sheath where the mean local potential is $\mathrm{V}_{\mathrm{eq}}=(-4.01 \pm 0.12) \mathrm{V}$. Its mean charge is $\mathrm{Q}_{\mathrm{d}}=(4.4 \pm 1.2) 10^{5} \mathrm{e}^{-}$. The error bar coming from the values dispersion given in Table 1 results in fact from the limited capacity of function (10) to represent al the experimental data.

Another method to estimate the charge consists in studying the vertical resonance oscillation of a dust particle, due to an initial excitation. By superimposition of 22 successive camera frames, the picture of Fig. 5 shows the vertical oscillation of a dust particle moving horizontally from the left to the right side, over the plate at the floating potential. In our experimental conditions, when many dust particles are trapped in a horizontal surface parallel to the plate, they are in liquid phase. Without excitation, their trajectories are rectilinear, without vertical or horizontal oscillations, with sudden direction changes in the horizontal plane, produced by collisions. To obtain vertical oscillations, we transmit an impulsion to the dust particles by suddenly decreasing the gas pressure. Then, to return to the initial conditions, the pressure is increased slowly in order to keep the local confining potential in the vertical direction $[39,40]$. In Fig. 5, the oscillation frequency is equal to $(17 \pm 1) \mathrm{Hz}$ and because the gas friction is negligible at the pressure of $10^{-3} \mathrm{mbar}$, we observe no damping of these oscillations. We can find the charge $\mathrm{Q}_{\mathrm{d}}$ writing the equilibrium equation in $\mathrm{z}$ direction [41]:

$$
m_{d} \ddot{z}+Q_{d} E-m_{d} g=0
$$

Assuming small oscillations around the equilibrium position $\mathrm{z}_{0}=(1.90 \pm 0.05) \mathrm{mm}$ and constant charge $\mathrm{Q}_{\mathrm{d}}$, Taylor expansion to first order of $\mathrm{E}$ in Eq. (11) provides: $\mathrm{Q}_{\mathrm{d}} \mathrm{E}=\mathrm{Q}_{\mathrm{d}} \mathrm{E}_{\mathrm{o}}+\mathrm{Q}_{\mathrm{d}} \mathrm{z}(\mathrm{dE} / \mathrm{dz})_{\mathrm{zo}}$. With the equilibrium condition: $Q_{d} E_{o}=m_{d} g$, the resonance frequency $\omega_{o}$ is:

$$
\omega_{o}^{2}=\frac{Q_{d}}{m_{d}}\left(\frac{d E}{d z}\right)_{z_{o}}
$$

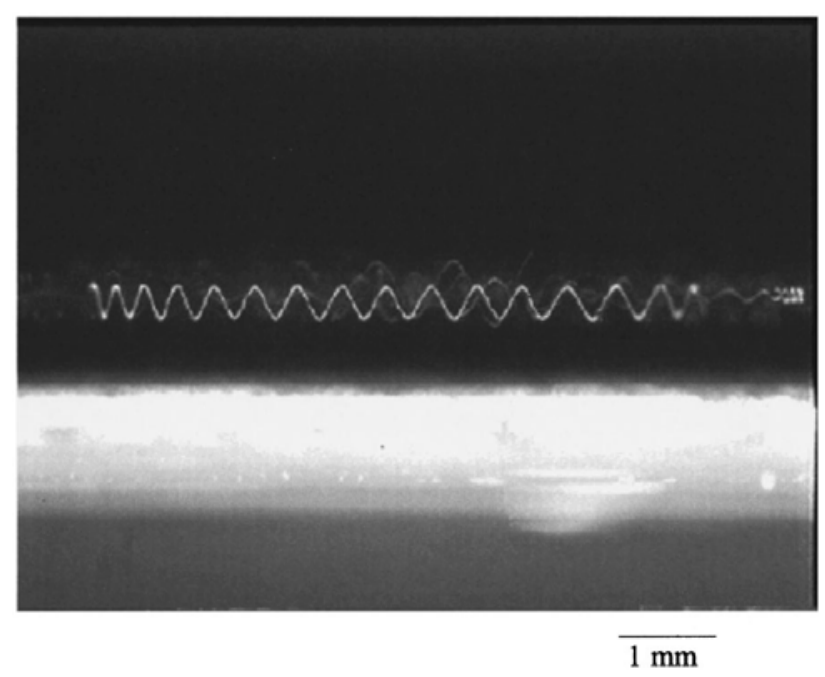

Fig. 5: Natural vertical oscillation of a dust particle levitating over a conducting plate while migrating from the left to the right side. This photo results from the superimposition of 22 successive camera frames (frame time duration: $0.04 \mathrm{~s}$ ). In this situation (argon pressure of $10^{-3} \mathrm{mbar}$ ) the dust particles trapped in the plate sheath edge are in a liquid phase. The frequency is equal to $(17 \pm 1) \mathrm{Hz}$ and the oscillation is not damped.

For $r_{d}=32 \mu \mathrm{m}$ and the electric field $\mathrm{E}$ deduced from Eq. (10) in the case where $V_{\text {bias }}=-26.5 \mathrm{~V}$, the dust charge is then: $\mathrm{Q}_{\mathrm{d}}=(4.3 \pm 0.5) 10^{5} \mathrm{e}^{-}$. The error bar is due to the uncertainty in the frequency measurement (the fit function being uniquely defined, it does not introduce any error in this case). We observe that this mean charge value is in good agreement with the one given by the levitation height measurements at $\mathrm{V}_{\text {bias }}=-26.5 \mathrm{~V}$. Indeed, both methods used the same potential profile. We see that this value is slightly higher than the one predicted by the OML model: $\left(\mathrm{Q}_{\mathrm{d}}\right)_{\mathrm{OML}}=(3.7 \pm 0.3) 10^{5} \mathrm{e}^{-}$. We assume that this higher numerical value originates from an underestimation of the electric field due, at least, to two facts. The first one is related to the use of a differential emissive probe diagnostic. An artificial flattening of potential profiles could be induced by this intrusive system, producing a decrease in the local electric field. The second one could be due to the appearance of a local sheath potential steepening in presence of dust particles. So, with our experimental measurements, in order to balance the gravitation effect, the dust particle charge must be higher. Nevertheless, the obtained experimental charge values again justify the neglect of the ion drag forces as shown using the dust charge calculated with the OML model.

\section{CONCLUSION}

Dust particles levitation experiments, in the sheath edge of a conducting horizontal disc plate embedded in a plasma produced by a dc discharge have been presented. The ionization sources consist of two heated tungsten filaments. The contribution of the 
energetic primary electrons emitted by the filaments has been taken into account for the high charge calculation of an isolated dust grain (OML model). Using a differential emissive probe diagnostic, we have measured the sheath-presheath plate profile for different negative plate biases. Then, using these potential profiles, we have calculated the forces applied to the dust particle. We show that the ion collection and ion coulomb drag forces are negligible. The charge of a dust particle is evaluated experimentally by two methods: i) by measuring the levitation height for each plate bias, corresponding approximately to the same sheath potential value and ii) through the dust particle natural vertical oscillation. In both cases, if we assume that the dust charge does not depend on its position in the plate sheath, we deduce an approximately constant value of the dust charge. The fact that the OML theory gives a value slightly lower than the two other methods could be explained by an underestimation of the measured electric field due to the perturbing differential emissive probe diagnostic and (or) by a change of the local sheath potential in presence of dust particles. This last point will be studied in a forthcoming publication.

\section{ACKNOWLEDGMENTS}

The authors would like to thank V. N. Tsytovich and S. V. Vladimirov for helpful discussions during their visits in Equipe Turbulence Plasma. The technical support of B. Squizzaro, K. Quotb and R. Bachelier is also greatly acknowledged. This work was supported by a grant from the Direction des Recherches et Etudes Techniques (DRET).

[1] J. P. Bøuf, Phys. Rev. A 46, 7910 (1992)

[2] A. Bouchoule, L. Boufendi, Plasma Sources Sci. Technol. 3, 293 (1994)

[3] N.N. Rao, P.K. Shukla, M.Y. Yu, Planet. Space Sci. 38, 543 (1990)

[4] R. Guerra, P.K. Shukla, Physica Scripta T63, 272 (1996)

[5] S. Benkadda, P. Gabbai, V.N. Tsytovich, A. Verga, Phys. Rev. E 53, 2717 (1996)

[6] U. Kortshagen, Appl. Phys. Lett. 71, 208 (1997)

[7] A. Barkan, R.L. Merlino, N. D’Angelo, Phys. Plasmas 2, 3563 (1995)

[8] J.B. Pieper, J. Goree, Phys. Rev. Lett. 77, 3137 (1996)

[9] M.S. Barnes, J.H. Keller, J.C. Foster, J.A. O’Neil, D.K. Coultas, Phys. Rev. Lett. 68, 313 (1992)

[10] M.D. Kilgore, J.E. Daugherty, R.K. Porteous, D.B. Graves, J. Appl. Phys. 73, 7195 (1993)

[11] H. Ikezi, Phys. Fluids 29, 1764 (1986)

[12] M.O. Robbins, K. Kremer, G.S. Grest, J. Chem. Phys. 88, 3286 (1988)

[13] V.N. Tsytovich, Physics Uspekhi 40, 53 (1997); V.N. Tsytovich, Y.K. Khodataev, R. Bingham, Comments Plasma Phys. Contr. Fusion 17, 221 (1996)

[14] S. Hamaguchi, R.T. Farouki, J. Chem. Phys. 101, 9876 (1994); R.T. Farouki, S. Hamaguchi, J. Chem. Phys. 101, 9885 (1994)
[15] F. Melandso, J. Goree, Phys. Rev. E 52, 5315 (1995)

[16] H. Totsuji, T. Kishimoto, C. Totsuji, Phys. Rev. Lett. 78, 3113 (1997)

[17] V.A. Schweigert, I.V. Schweigert, A. Melzer, A. Homann, A. Piel, Phys. Rev. Lett. 80, 5345 (1998)

[18] H. Thomas, G.E. Morfill, V. Demmel, Phys. Rev. Lett. 73, 652 (1994)

[19] A. Melzer, T. Trottenberg, A. Piel, Phys. Lett. A 191, 301 (1994)

[20] J.H. Chu, Lin I, Phys. Rev. Lett. 72, 4009 (1994)

[21] J.B. Pieper, J. Goree, R.A. Quinn, Phys. Rev. E 54, 5636 (1996)

[22] Y. Hayashi, K. Tachibana, J. Vac. Sci. Technol A 14, 506 (1996)

[23] V.E. Fortov, A.P. Nefedov, O.F. Petrov, A.A. Samarian, A.V. Chernyschev, A.M. Lipaev, JETP Lett. 63, 187 (1996)

[24] S. Nunomura, N. Ohno, S. Takamura, Phys. Plasmas 5, 3515 (1998)

[25] D.A. Law, W.H. Steel, B.M. Annaratone, J.E. Allen, Phys. Rev. Lett. 80, 4189 (1998)

[26] L. Boufendi, A. Bouchoule, R.K. Porteous, J.Ph. Blondeau, A. Plain, C. Laure, J. Appl. Phys. 73, 2160 (1993)

[27] J.L. Dorier, Ch. Hollenstein, A.A. Howling, J. Vac. Sci. Technol A 13, 918 (1995)

[28] R.N. Carlile, S. Geha, J.F. O’Hanlon, J. C. Stewart, Appl. Phys. Lett. 59, 1167 (1991)

[29] Y. Watanabe, M. Shiratani, M. Yamashita, Appl. Phys. Lett. 61, 1510 (1992)

[30] G.S. Selwyn, J. Singh, R.S. Bennett, J. Vac. Sci. Technol A 7, 2758 (1988)

[31] O. Havnes, T. Nitter, V. Tsytovich, G.E. Morfill, T. Harquist, Plasma Sources Sci. Technol. 3, 448 (1994)

[32] J. Perrin, P. Molinas-Mata, Ph. Belenguer, J. Phys. D: Appl. Phys. 27, 2499 (1994)

[33] I.B. Berstein, and I.N. Rabinowitz, Phys. Fluids 2, 112 (1959)

[34] M. Carrère, L. Chérigier, C. Arnas-Capeau, G. Bachet, F. Doveil, Rev. Sci. Instrum. 67, 4124 (1996)

[35] W. En Yao, T. Intrator, N. Hershkowitz, Rev. Sci. Instrum. 56, 519 (1985); N. Hershkowitz, IEEE Trans. Plasma Sci. 22, 11 (1994)

[36] B. Walch, M. Horanyi, S. Robertson, Phys. Rev. Lett. 75, 839 (1995)

[37] N. Meyer-Vernet, Astron. Astrophys. 105, 98 (1982)

[38] The rigorous expression of the capacitance is $\mathrm{C}=$ $4 \pi \varepsilon_{o} r_{d}\left(1+r_{d} / \lambda_{\mathrm{D}}\right)$ where the linearized Debye length $\lambda_{\mathrm{D}}$ is equal to the smallest value of the electrons or ion Debye length. Using the plate sheath potential shape, not yet presented in the text, we can calculate at each position from the plate, the electrons and ion Debye lengths whose amplitude are increasing as the distance from the plate decreases. At the levitation height, we find $\lambda_{\mathrm{D}} \sim \lambda_{\mathrm{Di}}$ where $\lambda_{\mathrm{Di}}$ is the ion Debye length and the ratio $r_{d} / \lambda_{\mathrm{Di}}$ is smaller than 1 .

[39] S.V. Vladimirov (private communication)

[40] S. Nunomura, T. Misawa, K. Asao, N. Ohno, S. Takamura, Proceedings of the Int. Conf. on Plasma Phys., Praha, June, 1998, ECA Vol. 22C, 2509 (1998)

[41] M. Zuzic, H.M. Thomas, G.E. Morfill, J. Vac. Sci. Technol. A 14, 496 (1996) 